\title{
Perceived cognitive function for breast cancer survivors: association of genetic and behaviorally related variables for inflammation
}

\author{
Jamie S. Myers ${ }^{1}$ - Theresa A. Koleck ${ }^{2}$ - Susan M. Sereika ${ }^{3}$ - Yvette P. Conley ${ }^{3}$. \\ Catherine M. Bender ${ }^{3}$
}

Received: 21 November 2016 / Accepted: 20 February 2017 / Published online: 1 March 2017

(C) Springer-Verlag Berlin Heidelberg 2017

\begin{abstract}
Purpose We explored relationships between genetic variability and behaviorally related variables (body mass index and exercise frequency) for inflammation, and perceived cognitive function (PCF) for breast cancer survivors (BCS). Our primary aim was to explore relationships between select singlenucleotide polymorphisms (SNPs) for ILIR1, IL6, TNF genes, and PCF. Our secondary aim was to explore whether body mass index (BMI) and exercise frequency moderate these relationships.

Methods We conducted an exploratory candidate gene substudy. Saliva samples from participants $(N=101)$ in a larger, cross-sectional study were genotyped. Multiple linear regression analysis was used to explore relationships between SNPs and PCF, controlling for age, education level, fatigue, and distress. Hierarchical expansion of regression models included main effects for BMI and exercise frequency and interaction effects between BMI, exercise frequency, and each SNP.

Results The most parsimonious regression model included fatigue, exercise frequency, and IL1RIrs2287047 minor alleles $(\mathrm{AA}+\mathrm{GG})\left(R^{2}=0.244\right.$, adjusted $R^{2}=0.220$, $p=0.013)$. No other SNPs were significant. Higher exercise frequency $(b=7.300, p=0.013$ ) and IL1RIrs2287047 (AA+ AG) $(b=6.512, p=0.025)$ predicted better PCF. Greater fatigue predicted poorer PCF $(b=-2.359, p<0.01)$. No
\end{abstract}

Jamie S. Myers

jmyers@kumc.edu

School of Nursing, University of Kansas, Kansas City, KS, USA

Columbia University, New York, NY, USA

3 School of Nursing, University of Pittsburgh, Pittsburgh, PA, USA interaction was demonstrated between BMI and exercise related to PCF or between BMI, exercise, and SNPs.

Conclusions Our results suggest a protective relationship between IL1R1rs2287047 (AA+AG) and PCF and provide further evidence supporting exercise as a potential intervention for poorer PCF. Ours is the first study to investigate genetic variability associated with inflammation, behaviorally related variables, and $\mathrm{PCF}$ for BCS.

Keywords Perceived cognitive function - Breast cancer . Genetics · Inflammation

\section{Introduction}

Cancer- and cancer treatment-related declines in cognitive function are highly prevalent and have a significant and deleterious effect on cancer survivors' quality of life [1]. Multiple mechanisms likely underlie these declines in cognitive function; among these are the inflammatory pathways associated with the development of cancer and cancer treatment [1]. Variability exists in the severity and duration of poorer cognitive function prior to adjuvant therapy and deterioration in cognitive function during and after treatment for breast cancer [1]. Genetic and behaviorally related factors for inflammation may influence this variability in cognitive function $[1,2]$. Research is needed to identify women who may be at increased risk for declines in cognitive function and, ultimately, to design appropriate interventions to prevent or mitigate the impact of cancer and cancer treatment on cognitive function. The purpose of our study was to explore the relationships between genetic variability and behaviorally related variables (body mass index and exercise frequency) for inflammation, and perceived cognitive function (PCF) for breast cancer survivors. 


\section{Background/significance}

During and shortly after chemotherapy for breast cancer, the incidence of declines in cognitive function is as high as $65 \%$ [3]. Compared to their healthy counterparts, up to $30 \%$ of women with breast cancer have poorer cognitive function prior to the initiation of chemotherapy, and long-term sequelae have been documented as long as 20 years after chemotherapy in this population [4]. Understanding the behavioral and genetic characteristics associated with the variability in the onset, severity, and duration of declines in cognitive function will further inform our knowledge of the causal mechanisms for cancer- and cancer treatment-related declines in cognitive function, identification of who is at risk for these problems, and the development of effective prevention and management strategies.

A number of biological pathways may be common to the process of natural aging as well as the development of cancerand cancer treatment-related declines in cognitive function, including both inflammation and genetic susceptibility [1, 5]. The release of pro-inflammatory cytokines such as interleukin-1 (IL1), interleukin-6 (IL6), and tumor necrosis factor alpha (TNF $\alpha$ ) is induced both by the body's inflammatory response to the tumor-related tissue invasion as well as to the administration of chemotherapy and has been associated with complaints of cognitive decline [6-9]. Variability in the expression of genes associated with these pro-inflammatory cytokines also may play a role in cognitive decline. Recent evidence suggests a relationship between polymorphisms of ILIRI and IL6 genes and participants' report of cognitive complaints $[10,11]$.

Obesity, or elevated body mass index (BMI), is associated with chronic inflammation (including higher levels of TNF $\alpha$ ) and a negative impact on the neural systems involved with cognition and memory [12-15]. Additionally, elevated BMI is associated with lower brain volumes and dementia risk in older-aged populations $[16,17]$. Women receiving chemotherapy for breast cancer typically gain weight during treatment which may further contribute to the development of cognitive declines and also is associated with increased risk of recurrence and progressive disease [18-21].

Exercise is known to mitigate cognitive decline in the elderly and may be important for women with breast cancer to maintain or reduce BMI and reduce the risk of recurrence and progressive disease $[22,23]$. Among the proposed mechanisms for the positive impact of exercise on cognitive function are decreases in inflammation, particularly in regions of the brain critical to executive function and memory (frontal lobe and hippocampus) [12, 24-26]. Preliminary work assessing the potential benefits of various forms of exercise as interventions for cancer-related declines in cognitive function has yielded encouraging results, such as decreased cognitive complaints and enhanced neuropsychological functioning
[27-29]. PCF is an individual's perception of their cognitive ability and is subjectively assessed through patients' self-report. Our own work demonstrated a negative association between BMI and PCF that was reduced by higher exercise frequency [2]. However, to our knowledge, no studies have been conducted to investigate the relationships between genetic variability for inflammation, behaviorally related variables (such as BMI and exercise patterns), and PCF for breast cancer survivors.

The primary aim of this study was to explore the relationships between genetic variability of select single-nucleotide polymorphisms (SNPs) for IL1 receptor 1 (ILIR1), IL6, and $T N F$ genes and PCF in women with breast cancer. The secondary aim was to explore whether BMI and exercise frequency moderate the relationships between the selected SNPs and PCF.

\section{Methods}

\section{Design and sample}

We conducted an exploratory candidate gene substudy participants who took part in a larger cross-sectional parent study [2]. The parent study was designed to investigate potential predictors of perceived cognitive impairment for women with breast cancer and included 363 participants ( 317 women with breast cancer and 46 controls). Eligible women were 18 years of age or older; able to read, write, and speak English; and with no history of previous malignancy, mental illness, dementia, or Alzheimer's disease. Parent study participants with breast cancer were diagnosed with any stage of disease. Participants with breast cancer were required to be scheduled for, receiving, or finished with chemotherapy including neoadjuvant therapy and were not excluded if currently receiving anti-estrogen therapy. Women with breast cancer were excluded for history of central nervous system metastases or myeloablative chemotherapy regimens in preparation for. The genetic substudy participants included only women with breast cancer who provided permission to be re-contacted following participation in the cross-sectional parent study $(N=143,39.4 \%)$. Of these, $101(70.6 \%)$ provided a saliva sample for DNA analyses.

\section{Dependent variable}

PCF was measured with the Functional Assessment of Cancer Therapy-Cognition Version 3 (FACT-COG) scale for perceived cognitive impairment (PCI, 18 items) [30]. PCI items are rated as to frequency of occurrence between 0 (never) and 4 (several times a day). Psychometric properties are established for the breast cancer population prior to and following chemotherapy (Cronbach's $\alpha=0.95, \mathrm{ICC}=0.82$ ) 
(Lynne Wagner, personal communication, 2/26/14). Higher scores indicate better perceived function.

\section{Predictor variables}

Genetic variants associated with inflammation included functional/putatively functional polymorphisms (i.e., SNPs) for IL6, IL1RI, and TNF (see Table 1). Selection of these SNPs was informed by the current literature. Tagging SNPs were identified through the use of the Phase III International HapMap project (archived data available at ftp://ftp.ncbi.nlm. nih.gov/hapmap/) [31, 32] and were selected using the following criteria: $R^{2} \geq 0.80$, minor allele frequency $>0.20$, and selected for Caucasian ancestry, which represents the majority of current sample.

Saliva samples were collected with Oragene DNA selfcollection kits from DNA Genotek Inc. (www.dnagenotek. com) and DNA was extracted from the saliva/buffer combination utilizing the protocol and reagents for extraction supplied with the Oragene kit [33]. The Sequenom iPLEX MassArray platform was used for genotyping (Sequenom Inc., San Diego, CA). SNPs with less than a 95\% call rate were re-genotyped using TaqMan allelic discrimination with the ABI Prism 7000 Sequence Detection System (SDS) and SDS software v1.2.3 (Thermo Fisher Scientific Inc., Waltham, MA) or eliminated from the analyses. Reliability evaluation steps included the following: double blind calls of genotypes, checks comparing expected homozygosity to observed homozygosity at each marker, checks of allele frequencies, genotype call rates, and checks of Hardy-Weinberg equilibrium (HWE).

$B M I$ was calculated from parent study participants' selfreport of height and weight. Self-report of height and weight commonly is used to calculate BMI due to the ease of data collection. Based on research results demonstrating minimal clinically relevant differences between self-reported and clinically measured height/weight, the accuracy of the continuous variable of BMI was within acceptable limits [34].

Exercise frequency was measured by parent study participants' self-report (none, monthly, 2-3 times/week, 4-5 times/ week, or daily). Due to sparsely populated categories, exercise frequency was dichotomized as an $(0,1)$ indicator variable (i.e., $0=\leq 2-3$ times/week and $1=\geq 4-5$ times/week) for regression analyses.

Well-known potential confounding factors or covariates included age (in completed years), education level (grade school, high school, college, graduate school), fatigue, and distress. Due to sparse data in the lower levels, education categories were collapsed, yielding a three-level education variable (high school or less, college, graduate school) from which two indicator variables were created for regression analyses, where graduate school was treated as the reference category. Fatigue and distress severity were assessed with the MD Anderson Symptom Inventory (MDASI) [35]. Participants rated MDASI items on a scale ranging from 0 (not present) to 10 (as bad as you can imagine). Cronbach's $\alpha$ for the MDASI ranges from 0.82 to 0.94 [35].

\section{Data analyses}

Data were analyzed with IBM SPSS Statistics version 23. Based on our earlier work, we determined a sample size of 100 achieves $80 \%$ power to detect effect sizes $\left(R^{2}\right)$ of 0.05 for individual SNPs with adjustment for a set of four additional variables (age, education, fatigue, and distress) explaining $R^{2}=0.24$ [2]. Differences between the substudy and parent sample were assessed with Pearson's chi-squared and $t$ tests.
Table 1 Minor allele frequency and $\operatorname{HWE}(N=101)$

\begin{tabular}{lllll}
\hline Gene & SNP & Minor allele & Percent & $\begin{array}{l}\text { HWE chi-squared } \\
\text { test } p \text { value }\end{array}$ \\
\hline IL1R1 & rs13020778 & $\mathrm{C}$ & 33.0 & 0.5565 \\
& rs3771200 & $\mathrm{A}$ & 32.0 & 0.9214 \\
& $\mathrm{rs391243}$ & $\mathrm{T}$ & 6.0 & $0.0457^{*}$ \\
& $\mathrm{rs3917304}$ & $\mathrm{T}$ & 27.0 & $0.0315^{*}$ \\
& $\mathrm{rs} 2287047$ & $\mathrm{~A}$ & 26.0 & 0.226 \\
& $\mathrm{rs} 949963^{\mathrm{a}}$ & $\mathrm{T}$ & 17.0 & $1.0000^{\mathrm{b}}$ \\
IL6 & $\mathrm{rs} 1800795^{\mathrm{a}}$ & $\mathrm{C}$ & 33.0 & 0.335 \\
IL6 & $\mathrm{rs} 2069840$ & $\mathrm{G}$ & 31.0 & 0.4033 \\
IL6 & $\mathrm{rs} 1474347$ & $\mathrm{C}$ & 33.0 & 0.1592 \\
TNF & $\mathrm{rs} 309366$ & $\mathrm{~T}$ & 20.0 & $0.1765^{\mathrm{b}}$ \\
\hline
\end{tabular}

$H W E$ Hardy-Weinberg equilibrium

$* p<0.05$

${ }^{\text {a }}$ Functional SNPs selected from the literature (Merriman et al. 2013; 2014)

${ }^{\mathrm{b}}$ Due to expected counts below five, Fisher's exact test was used instead of chi-squared test 
We calculated minor allele frequencies and assessed for departure from HWE. Standard descriptive statistics were computed based on each variable's level of measurement and observed distribution and data screening was conducted to detect any anomalies (e.g., outliers, missing data) that may impact the validity of results. Missing data was minimal and determined to be missing completely at random (MCAR) by Little's MCAR test [36]. Listwise deletion was used for all analyses. Bivariate correlations were calculated between the potential confounding variables (age, education, fatigue, and distress), the targeted predictor variables, and the dependent variable. Results of interest for both aims included the estimated regression coefficients and $95 \%$ confidence intervals for the unadjusted and adjusted regression coefficients. BMI and exercise frequency were incorporated into models both individually and collectively. Model assessment (residual analysis and assessment of potential influential observations in terms of estimation of predicted values, regression coefficients, and standard errors for regression coefficients) was performed for all fitted models. Although no univariate or multivariate outliers were identified through initial data screening, model assessment using residual analysis and influence diagnostics identified one participant to be influential (partial regression plots) across a number of variables (age, fatigue, distress, education, BMI, exercise frequency, and the ILIR1rs2287047 genotype). Through a sensitivity analysis, the bivariate correlations and regression model also were examined without the influential participant.

Primary aim analysis Multiple linear regression analysis was used to explore relationships between the presence of at least one copy of a minor allele (homozygous variant genotype or heterozygous genotype compared to homozygous wildtype genotype) for each identified SNP and the continuous outcome variable, PCF. First, models were fitted considering each SNP separately to estimate unadjusted regression coefficients with confidence intervals. To obtain adjusted estimators, the linear regression models were expanded in a hierarchical fashion to also include age and education level (first block), fatigue and distress as potential confounders/covariates (second block), and the allele of interest entered in the third block.

Secondary aim analysis In order to explore BMI and exercise frequency as possible moderators of the association of genetic variation on PCF, the multiple linear regression models for the selected SNPs were further expanded in a hierarchical fashion to also include main effects for BMI and exercise patterns in the fourth block and two-way interactions between BMI and exercise patterns with the presence of a minor allele in the fifth block. Interaction terms were created as the products of these variables. Post hoc regression analysis also was conducted to explore the potential impact of endocrine therapy.

\section{Results}

The genetic substudy sample $(N=101)$ mirrored the cancer survivors in the parent study sample as the majority were Caucasian, married, well-educated, post-menopausal women (see Table 2). The genetic substudy sample significantly differed from the parent sample for higher education levels $(p=0.045)$, lower stage disease $(p=0.015)$, lower BMI $(p=0.017)$, and less sleep disturbance $(p=0.031)$.

Genotyping was successful for 10 of the 12 identified SNPs. Two SNPs (IL1R1rs2160227 and IL1R1rs391265) were not successfully genotyped using either the iPLEX or TaqMan platforms. Minor allele frequency ranged from 6 to $37 \%$ (see Table 1). All SNPs were in HWE except two. We believe this departure from HWE is the result of our sample size and non-random (all women with breast cancer) sampling from the population.

Bivariate correlations between the PCF and the 10 individual SNPs indicated a trend toward significance for only one SNP (IL1R1rs2287047) $(r=0.177, p=0.080)$ (see Table 3). In our sample, 99 women were genotyped successfully for this polymorphism. Significant correlations were found between PCF and exercise frequency, fatigue, and distress (see Table 3). Fatigue and distress also were highly correlated (see Table 4). No significant correlations were found between BMI and PCF or BMI and exercise. No significant correlations were found between exercise and any of the SNPs.

Aim 1 Multiple linear regression modeling for each SNP and association with PCF were not significant. Model expansion to control for age, education, fatigue, and distress was significant for possession of one or more minor ILIR1rs2287047 alleles $(\mathrm{AA}+\mathrm{AG})$ compared to possession of the homozygous wildtype genotype (GG) $\left(R^{2}=0.204\right.$, adjusted $R^{2}=0.152$, $b=6.151, p=0.047$ ). The IL1R1rs2287047 (AA+AG) genotype was associated with better PCF.

Aim 2 Further model expansion to examine the main effects of BMI and exercise frequency on PCF was no longer significant for the full sample of genetic substudy participants $\left(R^{2}=0.237\right.$, adjusted $\left.R^{2}=0.170, p=0.145\right)$. However, fatigue ( $b=-1.888, p=0.007$ ) was a significant predictor of poorer PCF and both higher exercise frequency $(b=6.501$, $p=0.049955)$ and ILIRIrs2287047 (AA+AG) predicted better PCF ( $b=5.955, p=0.052)$ (see Table 4$)$. Neither age nor educational level were significant contributors to the model, nor were BMI or distress.

Results of the multiple linear regression yielded no significant interactions between BMI and PCI or BMI and exercise. Likewise, no significant interactions were found between exercise frequency and possession of IL1R1rs2287047 (AA+ $A G)$. 
Table 2 Sample description $(N=101)$

Age (mean, SD, minimum-maximum)

BMI (mean, SD, minimum-maximum)

Exercise frequency $(N, \%)$

None

Monthly

2-3 times/week

4-5 times/week

Daily

Menopause status $(N, \%)$

Pre

Peri

Post

Chemotherapy status

Pre

Current

Post

Endocrine therapy received $(N, \%)$

Radiation therapy received $(N, \%)$

Stage of breast cancer

I $(N, \%)$

II $(N, \%)$

III $(N, \%)$

IV $(N, \%)$

Not reported $(N, \%)$

Hispanic or Latino ethnicity $(N, \%)$

Race $(N, \%)$

Caucasian

African-American

Asian

Pacific Islander

Marital status $(N, \%)$

Single

Married

Separated

Divorced

Widowed

In a relationship

Education level $(N, \%)$

High school

College

Graduate school

Work status $(N, \%)$

FT homemaker

PT homemaker

FT work

PT work

Retired

Medical leave

Not working

PCI score (mean, SD, minimum-maximum)
$54.86,10.78,34-89$

$27.27,6.21,18-46$

24, 24.2

$4,4.0$

$28,28.3$

$28,28.3$

$15,15.2$

$12,11.9$

$5,5.0$

$84,83.2$

14, 13.9

11, 11.9

76, 75.2

51, 50.5

54, 53.5

$35,34.7$

46, 45.5

$10,9.9$

4, 4.0

6, 5.9

2, 2.0

95, 94.1

3, 3.0

2, 2.0

1, 1.0

8, 7.9

74, 73.3

2, 2.0

$6,5.9$

$6,5.9$

5, 5.0

13, 12.9

$60,59.4$

28, 27.7

11, 10.9

1, 1.0

$47,46.5$

19, 18.8

19, 18.8

2, 2.0

8, 7.9

$46.79,16.43,13-72$
Table 2 (continued)

\begin{tabular}{ll}
\hline Fatigue (mean, SD, minimum-maximum) & $3,2.68,0-10$ \\
Distress (mean, SD, minimum-maximum) & $1.88,2.52,0-10$ \\
Sleep disturbance & $2.49,2.63,0-9$ \\
(mean, SD, minimum-maximum) & \\
Depression (mean, SD, minimum-maximum) & $1.58,2.38,0-10$
\end{tabular}

$B M I$ body mass index, $S D$ standard deviation, $F T$ full time, $P C I$ perceived cognitive impairment, $P T$ part time

Analyses after omission of the influential case resulted in a significant bivariate correlation between IL1R1rs2287047 $(\mathrm{AA}+\mathrm{AG})$ and PCI $(.203, p=0.045)$ (see Table 3$)$ and the regression model explained $23 \%$ of the variance $\left(R^{2}=0.290\right.$, adjusted $R^{2}=0.226$ ). Possession of IL1R1rs2287047 (AA+ AG) contributed significantly $(b=6.592, p=0.024)$ to the model as did exercise frequency $(b=7.595, p=0.017$ ) (see Table 4). Age, education level, BMI, and distress remained non-significant in the model. Thus, the most parsimonious regression model included fatigue, exercise frequency, and ILIR1rs 2287047 genotype, explaining $22 \%$ of the variance $\left(R^{2}=0.242\right.$, adjusted $\left.R^{2}=0.220\right)$ (see Table 4$)$.

Controlling for endocrine therapy (ET) in the full regression model demonstrated significant bivariate correlation between ET and PCI $(-.288, p=0.004)$ (see Table 3). The revised full regression model explained $28 \%$ of the variance $\left(R^{2}=0.352\right.$, adjusted $\left.R^{2}=0.286\right)$. ET contributed significantly to the model $(b=-8.246, p=0.005)$ as did exercise frequency $(b=8.258, p=0.007)$ and $I L 1 R 1 r s 2287047$ (AA+AG) $(b=5.853, p=0.038)$. The parsimonious regression model explained $27 \%$ of the variance $\left(R^{2}=0.307\right.$, adjusted $R^{2}=0.277$ ) when controlling for ET.

\section{Discussion}

Possession of one or more IL1R1rs2287047 minor alleles $(\mathrm{AA}+\mathrm{AG})$ was associated with better PCF (i.e., higher PCI scores), suggesting a protective effect compared to possession of the homozygous wildtype genotype (GG). Higher levels of fatigue and distress were associated with worse perceived cognitive function (lower PCI scores). Higher levels of exercise frequency were associated with better PCF. We were unable to demonstrate interaction effects between BMI, exercise frequency, and genetic variability for inflammation.

The suggestion of a protective effect for possession of one or more IL1R1rs 2287047 minor alleles is intriguing. The $I L 1 R 1$ gene is located in a cluster of related cytokine receptor genes on chromosome 2q12. ILIRIrs2287047 is located in intron-1, a non-coding section of the gene. The A allele of this SNP is associated with a protective effect against severe osteoarthritis of the hand and, even though it is located on a noncoding section of the gene, was found to co-localize with predicted transcription factor binding sites [37]. The ILIRI 
Table 3 Correlations with PCI score for full sample and with influential case removed

\begin{tabular}{|c|c|c|c|c|c|c|}
\hline \multicolumn{7}{|c|}{ Correlations with PCI score for full sample $(N=99)$} \\
\hline & PCI score & SNP & $\begin{array}{l}\text { Exercise } \\
\text { frequency }\end{array}$ & Fatigue & Distress & $\begin{array}{r}\text { Endocrine } \\
\text { therapy }\end{array}$ \\
\hline PCI score & 1 & 0.177 & $0.207 *$ & $-0.370 * *$ & $-0.334 * *$ & $-0.260 * *$ \\
\hline Sig & & 0.080 & 0.038 & 0.000 & 0.001 & 0.009 \\
\hline IL1R1rs2287047-AA+AG & & 1 & 0.036 & 0.012 & 0.039 & -0.092 \\
\hline Sig & & & 0.722 & 0.903 & 0.704 & 0.368 \\
\hline Exercise frequency & & & 1 & 0.015 & $-0.213 *$ & 0.111 \\
\hline Sig & & & & 0.882 & 0.032 & 0.269 \\
\hline Fatigue & & & & 1 & $0.533 * *$ & 0.141 \\
\hline Sig & & & & & 0.000 & 0.160 \\
\hline Distress & & & & & 1 & 0.016 \\
\hline Sig & & & & & & 0.872 \\
\hline \multicolumn{7}{|c|}{ Correlations with PCI score with influential case removed $(N=98)$} \\
\hline & PCI score & SNP & $\begin{array}{l}\text { Exercise } \\
\text { frequency }\end{array}$ & Fatigue & Distress & $\begin{array}{r}\text { Endocrine } \\
\text { therapy }\end{array}$ \\
\hline PCI score & 1 & $0.203 *$ & $0.237 *$ & $-0.395 * *$ & $-0.349 * *$ & $-0.288 * *$ \\
\hline Sig & & 0.045 & 0.018 & 0.000 & 0.000 & 0.004 \\
\hline IL1R1rs2287047-AA+ & & 1 & 0.026 & 0.020 & 0.042 & -0.083 \\
\hline $\mathrm{AG}^{\mathrm{a}}$ & & & 0.802 & 0.848 & 0.680 & 0.419 \\
\hline \multicolumn{7}{|l|}{ Sig } \\
\hline Exercise frequency & & & 1 & 0.024 & $-0.211 *$ & 0.124 \\
\hline Sig & & & & 0.815 & 0.035 & 0.219 \\
\hline Fatigue & & & & 1 & $0.532 * *$ & 0.134 \\
\hline Sig & & & & & 0.000 & 0.182 \\
\hline Distress & & & & & 1 & 0.013 \\
\hline Sig & & & & & & 0.899 \\
\hline
\end{tabular}

$P C I$ perceived cognitive impairment, Sig significance

$* p<0.05 ; * * p<0.01$

gene is associated with activation of nuclear factor kappa $\mathrm{B}$, a modulator of inflammatory and immune gene expression that may be involved in the processes of synaptic plasticity and memory [38]. The IL1R1rs2287047 minor allele also is hypothesized to be protective against activation of the p38 pathway in response to stress stimuli and inflammatory signals [39]. The p38 mitogen-activated protein kinase (MAPK) signaling pathway is involved in cytokine production (including IL1 and TNF $\alpha$ ) and the stress response and has been implicated in postoperative pulmonary complications in lung cancer patients as well as other inflammatory processes such as Alzheimer's disease [40]. Further research with larger sample sizes is warranted to explore the potential protective effect for the IL1R1rs2287047 minor allele against complaints of decreased PCF for breast cancer survivors.

The association of higher exercise frequency with better PCF is consistent with previous research findings and provides further support of exercise as a potential intervention for complaints of decreased cognitive function in breast cancer survivors. Recently published results of two prospective studies demonstrated a relationship between polymorphisms of IL1R1rs949963, IL6rs1800795, and lower scores of selfreported attentional function for patients receiving radiation therapy for breast cancer and their caregivers $[10,11]$. We found no association between ILIRIrs 949963 , IL6rs 1800795 or TNF SNPs and PCF. This difference in findings may be due to our sample size, time since therapy completion, and the use of different measures of PCF.

Surprisingly, neither age nor education contributed significantly to the regression model. Participants' ages ranged from 34 to 89 years with a mean age of 54.86 years. The sample was not balanced in terms of education level. Only 13 participants were educated at less than college level. Measurement of years of education (as opposed to levels of education) may have been more informative. As expected, we found significant correlation between fatigue and distress, the other potentially confounding variables. Ultimately, fatigue was the only significant predictor when investigated in combination with exercise frequency and IL1R1rs2287047 genotype.

Model assessment identified one participant for whom our regression model did not generalize. Examination of the residual diagnostics indicated that this participant was influential across a number of independent variables (age, fatigue, distress, education, BMI, exercise frequency, and the IL1R1rs2287047 genotype). Interestingly, this participant was one of only two individuals of Hispanic ethnicity. She 
Table 4 Full regression model and parsimonious regression model with influential case removed

\begin{tabular}{|c|c|c|c|c|c|}
\hline \multicolumn{6}{|c|}{ Full regression model with influential case removed $(N=98) R^{2}=0.290$, adjusted $R^{2}=0.226$} \\
\hline & \multicolumn{3}{|c|}{ Unstd coefficients } & \multicolumn{2}{|l|}{$95 \%$ CI for $b$} \\
\hline Predictor & $b$ & SE & $p$ value & Lower bound & Upper bound \\
\hline (Constant) & 49.846 & 12.363 & & 25.281 & 74.410 \\
\hline Age (years) & 0.029 & 0.145 & 0.840 & -0.259 & 0.318 \\
\hline College $^{\mathrm{a}}$ & -4.778 & 3.315 & 0.153 & -11.364 & 1.808 \\
\hline High school $^{\mathrm{a}}$ & 2.766 & 5.005 & 0.582 & -7.179 & 12.711 \\
\hline Fatigue & -2.100 & 0.645 & 0.002 & -3.390 & -0.809 \\
\hline Distress & -0.819 & 0.715 & 0.256 & -2.024 & 0.603 \\
\hline BMI $\left(\mathrm{kg} / \mathrm{m}^{2}\right)$ & -0.014 & 0.251 & 0.956 & -0.513 & 0.485 \\
\hline Exercise frequency (4-5 days/week or more) ${ }^{\mathrm{b}}$ & 7.595 & 3.118 & .017 & 1.399 & 13.791 \\
\hline IL1R1rs2287047-AA+AG ${ }^{\mathrm{c}}$ & 6.592 & 20,874 & 0.024 & 0.882 & 12.302 \\
\hline \multicolumn{6}{|c|}{ Parsimonious regression model with influential case removed $(N=98) R^{2}=0.244$, adjusted $R^{2}=0.220$} \\
\hline & \multicolumn{3}{|c|}{ Unstd coefficients } & \multicolumn{2}{|l|}{$95 \% \mathrm{CI}$ for $b$} \\
\hline Predictor & $b$ & SE & $p$ value & Lower bound & Upper bound \\
\hline (Constant) & 48.025 & 2.824 & $\leq 0.001$ & 49.417 & 53.633 \\
\hline Fatigue & -2.359 & 0.533 & $\leq 0.001$ & -3.418 & -1.300 \\
\hline Exercise frequency (4-5 days/week or more $)^{\mathrm{b}}$ & 7.300 & 2.871 & $\leq 0.013$ & 1.600 & 13.000 \\
\hline IL1R1rs2287047-AA+AG ${ }^{c}$ & 6.512 & 2.850 & 0.025 & 0.854 & 12.170 \\
\hline
\end{tabular}

Unstd unstandardized, $S E$ standard error, $S I G$ significance, $C I$ confidence interval

${ }^{\text {a }}$ Graduate school was treated as the reference category for the education level

${ }^{\mathrm{b}} 2-3$ days/week or less treated as reference category

${ }^{\mathrm{c}}$ IL1R1rs2287047-GG was treated as reference category

was 42 years old with stage 1 disease and currently receiving chemotherapy at the time of her participation. She was highly educated (graduate school), premenopausal, married, employed full time, of normal weight (BMI 23), and exercised frequently (4-5 times/week). She reported low levels of fatigue and distress (ranked at 1 out of 10) and no pain or pain medications. Additional available data were examined to try to explain lack of model fit for this participant. Based on responses to the MDASI that we did not include in the regression model due to high correlation with fatigue and distress, we reviewed participants' ranking for depressive symptoms and disturbed mood. This participant reported a high level of depressive symptoms (ranked 9 out of 10) and disturbed mood (ranked 7 out of 10). Only one participant in the sample reported higher depression (10 out of 10). As with fatigue and distress, depression and mood disturbance are known to be potential confounders for PCF and are associated with markers of inflammation [1, 6, 41]. Our relatively small sample size and lack of diversity hampered our ability to further discern potential differences for this participant. In addition to depression, other genetic factors that may influence PCF would be of interest such as variation in DNA repair and oxidative stress. A larger, more diverse sample is needed for future research.

This genetic substudy had a number of limitations including a cross-sectional design and no objective measures of cognitive function. Prospective research including objective cognitive measures is needed. However, recent evidence has shown that patients' self-report of poorer cognitive function is confirmed by neuroimaging studies and actually precedes objectively measured cognitive deficits [42]. This evidence highlights the importance of self-reported changes in cognitive function as a study endpoint. Poorer PCF is associated with negative psychological well-being, decision-making ability, effectiveness in the workplace, and social relationships [43-46]. Lack of age- and education-matched controls limited our ability to distinguish the influence of disease on cognitive function in this analysis. Objective measurement of exercise frequency such as electronic physical activity monitoring would have provided a more sensitive and comprehensive assessment of physical activity.

Our study was exploratory. Thus, the sample size was somewhat small for genetic research. Additionally, the genetic substudy sample significantly differed from the parent sample in three areas: lower stage disease, higher education levels, and lower BMI. The lower BMI may have contributed to the inability to demonstrate an interaction between BMI and exercise frequency related to PCF in contrast to the parent study results. The small sample size may have contributed to the lack of interaction between exercise frequency and genetic variability and prevented us from controlling for the impact of time since completion of therapy. However, the results of 
this study clearly demonstrate the need for additional research to further explore the potential protective effect of exercise frequency and genetic variability, such as possession of the minor allele for IL1R1rs2287047 (AA+AG).

Our sample size also limited the number of potential covariates for control in our original data analysis plan. Estrogenic action has been implicated as a moderator of cognitive function. The impact of endocrine therapy on cognitive function for women with breast cancer has been mixed, although evidence has shown that tamoxifen and aromatase inhibition have been associated with decreased function in various cognitive domains $[47,48]$. About half of our sample $(N=51$, $51 \%)$ reported receiving endocrine therapy with tamoxifen $(N=35,34.7 \%)$ and/or one or more aromatase inhibitors $(N=43,42.6 \%)$. Our post hoc examination of the contribution of endocrine therapy supports previous evidence of a detrimental impact on PCF.

\section{Conclusions}

Our primary aim was to explore the relationship between genetic variability of select SNPs for IL1R1, IL6, and TNF associated with inflammation and PCF. Our results suggest a possible protective relationship between possession of the minor allele for IL1R1rs2287047 (AA+AG) and PCF.

Our secondary aim was to explore whether BMI and exercise frequency moderate the relationship between selected SNPs and PCF. In this genetic substudy, we were unable to demonstrate interactions between BMI, exercise frequency, and the SNPs associated with inflammation that were genotyped. However, as with our earlier work, higher exercise frequency was a significant predictor of better perceived cognitive function. Our results provide further support to the hypothesis that exercise frequency may mitigate the complaints of decreased cognitive function for survivors of breast cancer.

These data are intriguing and hypothesis generating. Further research with a larger and more ethnically diverse sample is needed to investigate potential interactions between ethnicity, depressive symptoms, and genetic phenotype. A larger sample size also is needed to further examine the relationship observed between BMI, exercise frequency, and cognitive function in our earlier work.

Acknowledgements Dr. Myers was a 2015 scholar and recipient of the American Nurses Foundation grant funded by the Jean E. Johnson PhD, RN, FAAN and Dorothy A. Cornelius Endowment Funds. Dr. Myers' time also was supported by the National Institution of Nursing Research (NINR) T32 and Interdisciplinary Training of Nurse Scientists in Cancer Survivorship while a postdoctoral scholar at the University of Pittsburgh School of Nursing.

Compliance with ethical standards Institutional Review Board (IRB) approvals were obtained and all participants signed informed consent prior to any data collection for the genetic substudy. All procedures performed in this study were in accordance with the ethical standards of the institutions and were performed in accordance with the ethical standards as laid down in the 1964 Declaration of Helsinki and its later amendments or comparable ethical standards. Only the participants' unique study number (associated with all study-related data) accompanied participants' genetic samples to the lab. All data analyses were conducted on deidentified information.

Conflict of interest Dr. Myers was a 2015 scholar and recipient of the American Nurses Foundation grant funded by the Jean E. Johnson PhD, RN, FAAN and Dorothy A. Cornelius Endowment Funds. Dr. Myers' time also was supported by the National Institution of Nursing Research (NINR) T32 and Interdisciplinary Training of Nurse Scientists in Cancer Survivorship while a postdoctoral scholar at the University of Pittsburgh School of Nursing.

\section{References}

1. Ahles TA, Root JC, Ryan EL (2012) Cancer- and cancer treatmentassociated cognitive change: an update on the state of the science. J Clin Oncol 30:3675-3686

2. Myers JS, Wick J, Klemp JR (2015) Potential factors associated with perceived cognitive impairment in breast cancer survivors. Support Care Cancer (ePub ahead of print). doi:10.1007/s00520015-2708-7

3. Wefel J, S AK, Buzdar AU, Meyers C (2010) Acute and late onset cognitive dysfunction associated with chemotherapy in women with breast cancer. Cancer 116:3348-3356. doi:10.1002/cncr. 25098

4. Koppelmans V, Breteler MM, Boogerd W, Seynaeve C, Schagen SB (2013) Late effects of adjuvant chemotherapy for adult onset non-CNS cancer; cognitive impairment, brain structure and risk of dementia. Clin Rev Oncol/Hematol 88:87-101

5. Mandleblatt JS, Hurria A, BC MD, Saykin A, Stern RA, JW VM, Mc Guckin M, Traina T, Denduluri N, Turner S, Howard D, Jacobsen PB, Ahles TA, Study TaLwC (2013) Cognitive effects of cancer and its treatments at the intersection of aging: what do we know, what do we need to know? Semin Oncol 40:709-725

6. Ahles TA, Saykin AJ (2007) Candidate mechanisms for chemotherapy-induced cognitive changes. Nat Rev Cancer 7(3): 192-201

7. Maier SF, Watkins LR (2003) Immune-to-central nervous system communication and its role in modulating pain and cognition: implications for cancer and cancer treatment. Brain Behav Evol 17(Supp 1.1):S235-S131

8. Niiya M, Niiya K, Kiguchi R, Shibakura M, Asaumi N, Shinagawa K, Ishimuru F, Kiura K, Ideda K, Ueoka H, Tanimoto M (2003) Induction of TNF-alpha, uPA, IL-8, and MCP-1 by doxorubicin in human lung cancer cells. Cancer Chemother Pharmacol 52:391398

9. Wood LJ, Nail LM, Gilster A, Winters KA, Elsea CR (2006) Cancer chemotherapy-related symptoms: evidence to suggest a role for proinflammatory cytokines. Oncol Nurs Forum 33:535-542

10. Merriman JD, Aouizerat BE, Cataldo JK, Dunn L, Cooper B, West C, Paul SM, Baggott CR, Dhruva A, Kober K, Langford DJ, Leutwyler H, Ritchie CS, Abrams G, Dodd M, Elboim C, Hamolsky D, Melisko M, Miaskowski C (2014) Association between an interleukin 1 receptor, type I promoter polymorphism and self-reported attentional function in women with breast cancer. Cytokine 65:192-201

11. Merriman JD, Aouizerat BE, Langford DJ, Cooper B, Baggott CR, Cataldo JK, Dhruva A, Dunn L, West C, Paul SM, Ritchie CS, 
Swift PS, Miaskowski C (2013) Preliminary evidence of an association between an interleukin 6 promoter polymorphism and selfreported attentional function in oncology patients and their familiy caregivers. Biol Res Nurs 16:152-159

12. Erion JR, Wosiski-Kuhn M, Dey A, Hao S, Davis CL, Pollock NK, Stranahan AM (2014) Obesity elicits interleukin 1-mediated deficits in hippocampal synaptic plasticity. J Neurosci 34:2618-2631

13. Hotamisligil GS, Shargill NS, Spiegelman BM (1993) Adipose expression of tumor necrosis factor-alpha: direct role in obesitylinked insulin resistance. Science 259(5091):87-91

14. Stienstra R, Duval C, Muller M, Kersten S (2007) PPARs, obesity, and inflammation. PPAR Res. doi:10.1155/2007/95974

15. Tzanetakou IP, Katsilambros NL, Benetos A, Mikhailidis DP, Perrea DN (2012) "Is obesity linked to aging?": adipose tissue and the role of telomeres. Ageing Res Rev 11:220-229

16. Ho AJ, Raji CA, Becker JT, Lopez OL, Kuller LH, Hua X, Lee S, Hibar D, Dinov ID, Stein JL, Jack CR, Weiner MW, Toga AW, Thompson PM (2010) Obesity is linked with lower brain volume in $700 \mathrm{AD}$ and MCI patients. Neurobiol Aging 31:1326-1339

17. Siervo M, Arnold R, Wells JCK, Tagliabue A, Colantuoni A, Albanese E, Brayne C, Stephan BCM (2011) Intentional weight loss in overweight and obese individuals and cognitive function: a systematic review and meta-analysis. Obes Rev 12:968-983

18. Lankester KJ, Phillips E, Lawton PA (2002) Weight gain during adjuvant and neoadjuvant chemotherapy for breast cancer: an audit of 100 women receiving FEC or CMF chemotherapy. Clin Oncol (R Coll Radiol) 14:64-67

19. Rock CL, Flatt SW, Newman V, Caan BJ, Haan MN, Stefanick ML, Faerber S, Pierce J (1999) Factors associated with weight gain in women after diagnosis of breast cancer. J Am Diet Assoc 99:1212 1221

20. da Cunha PA, de Carlos Back LK, Sereia AF, Kubelka C, Ribeiro MC, Fernandes BL, de Souza IR (2013) Interaction between obesity-related genes, FTO and MC4R, associated to an increase of breast cancer risk. Mol Biol Rep 40:6657-6664

21. Dalamaga M (2013) Obesity, insulin resistance, adipocytokines and breast cancer: new biomarkers and attractive therapeutic targets. World J Exp Med 3(3):34-42

22. Carvalho A, Rea IM, Parimon T, Cusack BJ (2014) Physical activity and cognitive function in individuals over 60 years of age: a systematic review. Clin Interv Aging 9:661-682

23. Scott E, Daley AJ, Doll H, Woodroofe N, Coleman RE, Mutrie N, Crank H, P JJ, Saxton JM (2013) Effects of an exercise and hypocaloric healthy eating program on biomarkers associated with long-term prognosis after early-stage breast cancer: a randomized controlled trial. Cancer Causes Control 24:181-191

24. Erickson KI, Gildengers AG, Butters MA (2013) Physical activity and neuroplasticity. Dialogues Clin Neurosci 15:99-108

25. Fardell JE, Vardy J, Shah JD (2012) Cognitive impairments caused by oxaliplatin and 5-fluorouracil chemotherapy are ameliorated by physical activity. Psychopharmacology 220:183-193

26. Noble EE, Mavanji V, Little MR, Billington CJ, Kotz CM, Wang C (2014) Exercise reduces diet-induced cognitive decilne and increases hippocampal brain-derived neurotrophic factor in CA3 neurons. Neurobiol Learn Mem (epub ahead of print). doi:10.1016/j. nlm.2014.04.006

27. Derry HM, Jaremka LM, Bennett JM, Peng J, Andridge R, Shapiro CL, Malarkey WB, Emery CF, Layman R, Mrozek EE, Glaser R, Kiecolt-Glaser JK (2014) Yoga and self-reported cognitive problems in breast cancer survivors: a randomized controlled trial. Psychooncology. doi:10.1002/pon.3707

28. Marinac CR, Godbole S, Kerr J, Natarajan L, Patterson RF, Hartman SJ (2015) Objectively measured physical activity activity and cognitive functioning in breast cancer survivors. J Cancer Surviv 9:230-238
29. Oh B, Butow PN, Mullan BA, Clarke SJ, Beale PJ, Pavlakis N, Lee MS, Rosenthal DS, Larkey L, Vardy J (2012) Effect of medical qigong on cognitive function, quality of life, and a biomarker of inflammation in cancer patients: a randomized controlled trial. Support Care Cancer 20:1235-1242. doi:10.1007/s00520-0111209-6

30. Wagner L, Sweet J, Butt Z, Lai J, Cella D (2009) Measuring patient self-reported cognitive function: development of the functional assessment of cancer therapy-cognitive function instrument. J Support Oncol 7:W32-W39

31. Gibbs RA, Belmont JW, Hardenbol P, Willis TD, Yu F, Yang H, Ch'ang L-Y, Huang W, Liu B, Shen Y (2003) The international HapMap project. Nature 426:789-796

32. Patnala R, Clements J, Batra J (2013) Candidate gene association studies: a comprehensive guide to useful in silico tools. BMC Genet 14. doi:10.1186/1471-2156-14-39

33. Laboratory protocol for manual purification of DNA from whole sample. (2012) DNA Genotek Inc.

34. Craig BM, Adams AK (2009) Accuracy of body mass index categories based on self-reported height and weight among women in the United States. Matern Child Health J 13:489-496

35. Cleeland CS, Mendoza TR, Wang XS, Chou C, Harle MT, Morrissey M, Engstrom MC (2000) Assessing symptom distress in cancer patients: the M.D. Anderson symptom inventory. Cancer 89:1634-1646

36. Little RJ (1998) A test of missing completely at random for multivariate data with missing values. J Am Stat Assoc 83:1198-1202

37. Nakki A, Kouhia ST, Saarela J, Harilainen A, Tallroth K, Videman T, Battie MC, Kaprio J, Peltonen L, Kujala UM (2010) Allelic variants of IL1R1 gene associate with severe hand osteoarthritis. BMC Med Genet 11:50. doi:10.1186/1471-2350-11-50

38. Albensi BC, Mattson MP (2000) Evidence for the involvement of TNF and NF-kappaB in hippocampal synaptic plasticity. Synapse 35(2):151-159. doi:10.1002/(sici)1098-2396(200002)35:2<151:: aid-syn8 $>3.0$. co;2-p

39. Hildebrandt MA, Roth JA, Vaporciyan AA, Pu X, Ye Y, Correa AM, Kim JY, Swisher SG, Wu X (2015) Genetic variation in the TNF/TRAF2/ASK1/p38 kinase signaling pathway as markers for postoperative pulmonary complications in lung cancer patients. Sci Rep 5:12068. doi:10.1038/srep12068

40. Kitazawa M, Cheng D, Tsukamoto MR, Koike MA, Wes PD, Vasilevko V, Cribbs DH, LaFerla FM (2011) Blocking IL-1 signaling rescues cognition, attenuates tau pathology, and restores neuronal beta-catenin pathway function in an Alzheimer's disease model. J Immunol 187(12):6539-6549. doi:10.4049/jimmunol.1100620

41. Myers JS (2008) Proinflammatory cytokines and sickness behavior: implications for depression and cancer-related symptoms. Oncol Nurs Forum 35:802-807

42. Saykin A, Wishart HA, Rabin LA, Santulli RB, Flashman LA, West JD, McHugh TL, Mamourian AC (2006) Older adults with cognitive complaints show brain atrophy similar to that of amnestic MCI. Neurology 67:834-842

43. Bender CM, Paraska KK, Sereika SM, Ryan CM, Berga SL (2001) Cognitive function and reproductive hormones in adjuvant therapy for breast cancer: a critical review. J Pain Symptom Manag 21:407424

44. Boykoff N, Moleni M, Subramanian SK (2009) Confronting chemobrain: an in-depth look at survivors' reports of impact on work, social networks, and health care response. J Cancer Surviv $3: 223-232$

45. Myers JS (2012) Chemotherapy-related cognitive impairment: the breast cancer experience. Oncol Nurs Forum 39:E31-E40. doi:10. 1188/12.OONF.E31-E40

46. Von Ah D, Haberman B, Carpenter JS, Schneider BL (2012) Impact of perceived cognitive impairment in breast cancer survivors. European Journal of Oncology Nursing 
47. Bender CM, Merriman JD, Gentry AL, Ahrendt GM, Berga SL, Brufsky AM, Casillo FE, Dailey MM, Erickson KI, Kratofil FM, McAuliffe PF, Rosenzweig MQ, Ryan CM, Sereika SM (2015)

Patterns of change in cognitive function with anastrozole therapy. Cancer 121(15):2627-2636. doi:10.1002/cncr.29393
48. Ganz PA, Petersen L, Castellon SA, Bower JE, Silverman DH, Cole SW, Irwin MR, Belin TR (2014) Cognitive function after the initiation of adjuvant endocrine therapy in early-stage breast cancer: an observational cohort study. J Clin Oncol 32(31):3559-3567. doi:10. 1200/jco.2014.56.1662 